\title{
ARTICLE
}

\section{Associations between eating disorders diagnoses, behaviours and menstrual dysfunction in a clinical sample}

\author{
Maria Giulia Martini ${ }^{1,2}$, Francesca Solmi ${ }^{3}$, Isabel Krug ${ }^{4}$, Andreas Karwautz ${ }^{5,}$ Gudrun Wagner ${ }^{5}$, \\ Fernando Fernandez-Aranda ${ }^{6}$, Janet Treasure ${ }^{7}$, Nadia Micali ${ }^{1,8}$ \\ 1. Institute of Child Health, Behavioral and Brain Sciences Unit, University College London, 30 \\ Guilford Street, WC1N 1EH, London, UK \\ 2. Psychiatric Unit, United Hospitals of Ancona and Academic Department of Experimental and \\ Clinical Medicine, Polytechnic University of Marche, via Conca 71, Ancona, Italy
}

3. Department of Applied Health Research, University College London, 1-19 Torrington Place, London, WC1E 7HB, UK

4. School of Psychological Sciences, University of Melbourne, Melbourne, Australia

5. Department of Child and Adolescent Psychiatry, Medical University of Vienna, Währinger Gürtel 18-20, 1090, Vienna, Austria

6. Department of Psychiatry, University Hospital Bellvitge-IDIBELL and CIBER Fisiopatología Obesidad y Nutrición (CIBERobn), Barcelona, Spain

7. Eating Disorders Research Unit, Psychological Medicine, Institute of Psychiatry, King's College London, London, UK

8. Dept. of Psychiatry, Icahn School of Medicine at Mount Sinai, New York, USA

\begin{abstract}
We explored associations between lifetime eating disorder (ED) diagnoses and behaviors and menstrual dysfunction using logistic regression models. Body mass index (BMI) fully explained differences in the odds of secondary amenorrhea (SA) across diagnoses. Women with dieting behaviors had borderline significantly higher odds of SA than those without after accounting for BMI. We suggest the presence of a strong association between BMI and SA and that dieting might represent a risk factor for SA regardless of BMI and ED diagnosis.
\end{abstract}

\section{Keywords}

Eating disorders, Amenorrhea, Menstrual dysfunctions, Bodymass index, Dieting

Menstrual Dysfunction (MD) is frequent in women with eating disorders (ED) and, particularly in women with Anorexia Nervosa (AN)(Algars et al. 2014). Although no longer a diagnostic criterion for the latter in the Diagnostic and Statistical Manual of mental disorders $5^{\text {th }}$ Edition (DSM5), amenorrhea is one of its most common physical correlates(Algars et al. 2014). An increasing body of literature(Poyastro Pinheiro et al. 2007) has shown that amenorrhea and oligomenorrhea can also occur in women with Bulimia Nervosa (BN). In this group, however, evidence is inconsistent, with studies showing a prevalence of secondary amenorrhea in $7-40 \%$ and of oligomenorrhea in 
37-64\% of cases, respectively(Poyastro Pinheiro et al. 2007; Gendall et al. 2000). Although the literature suggests that malnutrition-related impairments in the hypothalamic-pituitary-ovarian axis and Polycystic ovary syndrome (PCOS) could be risk factors for developing secondary amenorrhea in women with $\mathrm{AN}$ and BN, respectively(Vyver et al. 2008) evidence is still inconclusive with respect to both its prevalence across diagnoses and risk factors/mechanisms.

ED behaviours such as binge-eating, purging, dieting, and excessive exercising have also been associated with MD independent of ED diagnoses(Poyastro Pinheiro et al. 2007; Austin et al. 2008; Gendall et al. 2000). Energy intake fluctuations typical of binge-eating, could affect insulinresistance, whereas raised insulin secretion and opioid and dopamine release resulting from purging behaviours could cause impairments of menstrual function(Tortorella et al. 2014). Although impairments in gonadotropin secretion following weight loss, often associated with dieting and exercising(Montero et al. 1996; Mendelsohn and Warren 2010) have been implicated in the onset of $\mathrm{MD}$, evidence on the role of low Body Mass Index (BMI), often associated with these behaviours, in causing menstrual irregularities is contradicting(Vyver et al. 2008). Some studies suggest that low BMI is the only predictor of MD in women with ED(Dalle Grave et al. 2008). Others, however, have shown that amenorrhea often precedes weight loss and persists after weight recovery(Brambilla et al. 2003), or is independent from weight loss(Gendall et al. 2000) suggesting that other factors, such as neuroendocrine and psychological aspects (i.e. the persistence of anorexia-related symptoms such as body dissatisfaction) could be involved in the pathogenesis of amenorrhea(Brambilla et al. 2003).

Considering the scarcity of research in this area, we aimed to investigate: the association between MD (i.e. primary and secondary amenorrhea) and (a) AN [AN-Restrictive (AN-R) and ANBinge Purging (AN-BP)] and BN, and (b) ED behaviours, such as dieting, binge eating, purging and excessive exercising in a clinical ED sample (irrespective of diagnosis). Moreover, we explored the role of lowest ever BMI as a mediator of the association between ED and MD.

\section{Methods}

\section{Sample}

A total of 320 ED patients recruited from secondary and tertiary services and self-help groups in four European countries (UK, Austria, Spain, Slovenia) as part of a multicenter study(Krug et al. 2012). Ethics committees of all participating organisations approved the study and women gave their informed consent.

\section{Measures}

All variables were obtained through the EATATE diagnostic interview(Anderluh 2003), a purposelydesigned European adaptation of the Longitudinal Interval Follow-up Evaluation (LIFE)(Keller 1987), as it elicits information on frequency and duration of ED behaviours (e.g. dieting, bingeeating and purging), cognitions (e.g. fear of fatness, preoccupation with food, and weight concerns) and menstrual function. We derived DSM-IV lifetime diagnoses of AN [restrictive $(A N-R)$ or bingepurge type(AN-BP)] defined broadly as meeting all diagnostic criteria except amenorrhea, and BN, using a hierarchical approach. A diagnosis of AN-R 'trumped' other diagnoses based on the assumption of a recognized association with $\mathrm{MD}$ (Brambilla et al. 2003). The $A N-R$ group ( $\mathrm{N}=115)$ thus also included women who had crossed over to AN-BP $(\mathrm{N}=20 ; 17.4 \%)$ or $\mathrm{BN}(\mathrm{N}=16 ; 13.9 \%)$; $A N-B P(N=93)$ women included those who had crossed over to $\mathrm{BN}(\mathrm{N}=30 ; 32.3 \%)$, but not $\mathrm{AN}-\mathrm{R}$; whereas $B N$ : included women who only ever had $B N(N=43)$. Participants with eating disorder not otherwise specified (EDNOS) $(\mathrm{N}=12)$ and Binge Eating Disorder (BED) $(\mathrm{N}=13)$ were excluded due to small numbers.

To investigate our second aim, we employed each of the following lifetime behaviours (i.e. yes/no) as predictors (not mutually exclusive): (i) binge-eating $(n=145)$;(ii) dieting $(n=220)$; (iii) excessive exercise for weight-loss ( $n=169$ ); (iv)purging (self-induced vomiting, laxatives or diuretics) ( $n=169)$; (v) binge-eating and purging $(n=135)$. 
Lifetime menstrual function was defined as: normal, primary amenorrhea (period never started or menarche after 16 years), secondary amenorrhea (menstruation cycles ceased for $\geq 3$ months after menarche).

Other covariates employed were: age at interview, lifetime contraceptive use, age of onset of ED symptoms, and lifetime lowest BMI at final height.

Data analyses

The distribution of exposures, outcomes and covariates in the sample was described using crosstabulations with Fisher's exact test and ANOVAs.

We investigated the association between lifetime ED diagnoses, ED behaviours, and BMI and MD using crude and adjusted multinomial logistic and logistic regression models. A minimally adjusted model included: age, contraceptive use, and ED age of onset, as a priori confounders. Lowest lifetime BMI was subsequently included in a multivariate fully adjusted model to investigate its mediating role on the association between ED diagnosis, ED behaviours and MD.

Only participants with complete data on predictors and outcomes were included in the analyses ( $\mathrm{N}=247)$. A total of $5.6 \%, 10.9 \%, 6.6 \%$ and $1.6 \%$ women had missing data respectively on age, age of ED onset, contraceptive use, and lowest BMI. These data were imputed using multiple imputations with chained equations since the assumption of missing at random appeared plausible (Table S3). Analyses were run using Stata13.

\section{Results}

Sample

Our sample consisted of 247 ED participants (AN-R: $N=115$; $B N$ : $N=39$; AN-BP: $N=93$ ). Lifetime lowest BMI differed across diagnoses and ED behaviours (Table S1).

\section{ED diagnoses and MD}

Both primary and secondary amenorrhea were more prevalent in women with $\mathrm{AN}-\mathrm{R}(47.1 \%$ and $12.7 \%$, respectively) compared to women with other diagnoses (Table S2).

Women with BN had lower odds of lifetime secondary amenorrhea than women with AN-R in crude and minimally adjusted, but not in fully adjusted analyses (Table 1). Lifetime lowest BMI was associated with secondary amenorrhea both in crude and adjusted models OR=0.7 (95\% Cl:0.6$0.8)$.

\section{ED behaviours and MD}

Women with lifetime dieting behaviours and lifetime excessive exercising had higher odds of reporting secondary amenorrhea compared to those who did not in unadjusted and minimally adjusted models. In the fully adjusted models dieting was weakly associated with MD $(p<0.08)$, whilst excessive exercise was not. Women with lifetime binge-purge behaviours had lower odds of primary amenorrhea in crude and minimally adjusted, but not in fully adjusted models (Table 2).

\section{Discussion}

This study investigated the association between lifetime ED diagnoses and behaviours, and MD. Women with BN had lower odds of secondary amenorrhea compared to those with AN, and this difference was explained by lifetime lowest BMI. We did not find associations between specific ED diagnoses and primary amenorrhea. Across the overall sample, lifetime dieting was associated with secondary amenorrhea, mostly due to low lifetime BMI.

In line with previous studies showing that secondary amenorrhea is highly prevalent in women with broadly defined AN(Poyastro Pinheiro et al. 2007) we found that women with AN (R and BP) had a higher prevalence and odds of secondary amenorrhea compared to BN. This difference was 
fully explained by lower BMI in this group. Although our study cannot provide evidence that factors other than BMI, such as ED-associated psychopathology(Brambilla et al. 2003) (i.e. anorexiarelated symptoms such as drive of thinness) or comorbid behaviours(Gendall et al. 2000), could explain higher prevalence of amenorrhea in women with ED compared to healthy controls, it suggests that BMI is a strong discriminant across ED groups.

Evidence from ED samples suggests that ED behaviours (i.e. bingeing, purging, dieting, and excessive exercising) are associated with higher prevalence of secondary amenorrhea(Poyastro Pinheiro et al. 2007). In this study, we only found dieting to be associated with secondary amenorrhea independent of BMI, an association seen also in non-ED populations, independent of BMI(Montero et al. 1996). Absolute weight loss and the decrease of fat mass secondary to dieting could contribute to ovarian dysfunction leading to $M D$ (Vyver et al. 2008). More research is warranted to explore risk mechanisms as well as the associations across ED behaviours and a wider range of MD.

The main strength of this study was that ED diagnoses were obtained using a structured interview and included comprehensive lifetime data across a range of behaviours and cognitions. However, some limitations should be mentioned. We did not have a healthy control group; therefore, we could not compare the prevalence of MD in women with and without ED. The small size of index groups could have resulted in type II error, especially in analyses for primary amenorrhea, due to low power. We could not control for PCOS, thought to increase the risk of MD in women with binge-eating behaviours(Vyver et al. 2008). We were unable to establish the temporality of occurrence of exposure and outcomes. Further detail on timing of diagnosis and MD could be useful especially in women with cross-over diagnoses. We assessed behaviours irrespective of frequency, thus we could not investigate potential dose-response effects on outcomes.

In conclusion, in a clinical ED sample we found that low BMI was the factor most strongly associated with secondary amenorrhea, although dieting behaviours were associated with the latter irrespective of ED diagnosis. More research is warranted to investigate risk factors for MD in population-based samples and to understand biological mechanisms. Our findings suggest that clinicians should screen women presenting with weight loss or dieting for adverse reproductive outcomes.

\section{Acknowledgments}

Financial support was received from the European Union (Framework-V Multicenter Research Grant, QCK1-1999-916). CIBERobn is an initiative of ISCIII.

We would like to thank Dr Marija Anderluh for providing data from Slovenia.

\section{References}

Algars M, Huang L, Von Holle AF, Peat CM, Thornton LM, Lichtenstein P, Bulik CM (2014) Binge eating and menstrual dysfunction. Journal of psychosomatic research 76(1):19-22

Anderluh M (2003) Childhood Obsessive-Compulsive Personality Traits in Adult Women With Eating Disorders: Defining a Broader Eating Disorder Phenotype. American Journal of Psychiatry 160(2): 242

Austin S, Ziyadeh NJ, Vohra S, Forman S, Gordon CM, Prokop LA, Keliher A, Jacobs D (2008) Irregular Menses Linked to Vomiting in a Nonclinical Sample: Findings from the National Eating Disorders Screening Program in High Schools. J Adolesc Health 42(5): 450-457 
Brambilla F, Monteleone P, Bortolotti F, Dalle Grave R, Todisco P, Favaro A, Santonastaso P, Ramacciotti C, Paoli R, Maj M (2003) Persistent amenorrhoea in weight-recovered anorexics: psychological and biological aspects. Psychiatry research 118(3): 249-57

Dalle Grave R, Calugi S, Marchesini G (2008) Is amenorrhea a clinically useful criterion for the diagnosis of anorexia nervosa?. Behaviour research and therapy 46(12):1290-4

Gendall K, Gendall KA, Bulik CM, Joyce PR, McIntosh VV, Carter FA (2000) Menstrual cycle irregularity in bulimia nervosa. Associated factors and changes with treatment. Journal of psychosomatic research 49(6):409-15

Keller MB (1987) The Longitudinal Interval Follow-up Evaluation. Archives of General Psychiatry 44(6): 540

Krug I, Penelo E, Fernandez-Aranda F, Anderluh M, Bellodi L, Cellini E, di Bernardo M, Granero R, Karwautz A, Nacmias B, Ricca V, Sorbi S, Tchanturia K, Wagner G, Collier D, Treasure J (2012) Low social interactions in eating disorder patients in childhood and adulthood $\square: A$ multi-centre European case control study. Journal of Health Psychology 18(1):26-37

Mendelsohn F, Warren MP (2010) Anorexia, bulimia, and the female athlete triad: evaluation and management. Endocrinology and metabolism clinics of North America 39(1):155-67

Montero P, Bernis C, Fernandez V, Castro S (1996) Influence of body mass index and slimming habits on menstrual pain and cycle irregularity. Journal of Biosocial Science 28(03):315-323

Poyastro Pinheiro A, Thornton LM, Plotonicov KH, Tozzi F, Klump KL, Berrettini WH, Brandt H, Crawford S, Crow S, Fichter MM, Goldman D, Halmi KA, Johnson C, Kaplan AS, Keel P, LaVia M, Mitchell J, Rotondo A, Strober M, Treasure J, Woodside DB, Von Holle A, Hamer R, Kaye WH, Bulik CM. (2007) Patterns of menstrual disturbance in eating disorders. The International journal of eating disorders 40(5):424-34

Tortorella A, Brambilla F, Fabrazzo M, Volpe U, Monteleone AM, Mastromo D, Monteleone P (2014) Central and peripheral peptides regulating eating behaviour and energy homeostasis in anorexia nervosa and bulimia nervosa: A literature review. European Eating Disorders Review 22(5):307-320

Vyver E, Steinegger C, Katzman DK (2008) Eating disorders and menstrual dysfunction in adolescents. Annals of the New York Academy of Sciences 1135: 253-64 